\title{
Kollaboration mit Extended-Reality-Systemen - eine Kategorisierung
}

\author{
Peter Schopf $(\mathbb{D} \cdot$ Julia M. Jonas
}

Eingegangen: 11. Juli 2021 / Angenommen: 17. November 2021 / Online publiziert: 16. Dezember 2021

(C) Der/die Autor(en) 2021

Zusammenfassung Die Technologien Augmented Reality (AR) und Virtual Reality (VR), die mit dem Überbegriff Extended Reality (XR) zusammengefasst werden, beeinflussen die Möglichkeiten der Kommunikation und Kollaboration grundlegend. Sie können intuitiv als Schnittstelle diverser Informationsquellen genutzt werden und bieten neue Arten der Informationsdarstellung. Extended-Reality-Systeme (XRS) bauen auf diesen beiden Technologien, AR und VR, auf und haben großes Potenzial für ein breites Spektrum an Anwendungsfällen, insbesondere für Kollaborationen.

Trotz dieses Potenzials ist bisher oft nicht klar, wann, wo und wie XRS sinnvoll eingesetzt werden können. Dies verzögert Innovationen und die Einführung in Arbeitsprozesse. Bei Entscheidern ist die Abgrenzung zwischen AR und VR teilweise unzureichend bekannt bzw. es fehlt die Terminologie, um die unterschiedlichen Systeme adäquat zu beschreiben. An dieser Stelle setzt diese konzeptuelle Arbeit an. Sie hat zum Ziel, das Verständnis der Anwendungsmöglichkeiten von XRS für Kollaborationen zu erleichtern sowie bei der Terminologie zu unterstützen. Aufbauend auf einer zentralen Taxonomie aus dem Bereich, Computer-Supported Cooperative Work' (CSCW) wird eine Kategorisierung von XRS für Kollaborationen entwickelt. Ein Vergleich mit bereits bestehenden Taxonomien zeigt die Notwendigkeit einer

Peter Schopf $(\bowtie)$

Lehrstuhl für Wirtschaftsinformatik, insb. Innovation und Wertschöpfung,

Friedrich-Alexander-Universität Erlangen-Nürnberg (FAU), Lange Gasse 20, 90403 Nürnberg,

Deutschland

E-Mail: peter.schopf@fau.de

Siemens Industry Software GmbH, Am Kabellager 9, 51063 Köln, Deutschland

Julia M. Jonas

Center for Leading Innovation \& Cooperation (CLIC), HHL Leipzig Graduate School of

Management, Jahnallee 59, 04109 Leipzig, Deutschland

Siemens AG, Digital Industries, Siemenspromenade 1, 91058 Erlangen, Deutschland 
praktisch anwendbaren und unkompliziert handhabbaren Kategorisierung für die Praxis.

Diese Arbeit trägt durch Kategorisierung, Terminologie und Illustrationen, zu einem besseren Verständnis von XRS-Einsatzmöglichkeiten bei. Dazu werden Illustrationen aus dem Umfeld von industriellen Baustellen genutzt.

Schlüsselwörter Extended Reality · Computer-Supported Cooperative Work $(\mathrm{CSCW}) \cdot$ Augmented Reality $\cdot$ Virtual Reality $\cdot$ Kollaboration

\title{
Collaboration with Extended Reality Systems - a Categorization
}

\begin{abstract}
The technologies Augmented Reality (AR) and Virtual Reality (VR), which are summarized under the umbrella term Extended Reality (XR), fundamentally influence the possibilities of communication and collaboration. They can be used intuitively as an interface between various sources of information and offer new ways of presenting information. Extended Reality Systems (XRS) build on these two technologies, AR and VR, and have great potential for a wide range of use cases, especially for collaboration.

Despite this potential, it is often not yet clear when, where and how XRS can be used sensibly. This delays innovations and the introduction to work processes. The distinction between AR and VR is sometimes insufficiently known among decisionmakers or the terminology is missing to adequately describe the different systems. This is where this conceptual work begins. Its aim is to facilitate the understanding of the possible uses of XRS for collaborations and to support terminology. Based on a central taxonomy from the area of Computer-Supported Cooperative Work (CSCW), a categorization of XRS for collaborations is developed. A comparison with already existing taxonomies shows the necessity of a practically applicable and uncomplicated manageable categorization in practice.

This work contributes to a better understanding of XRS application possibilities through categorization, terminology and illustrations. For this purpose, illustrations from the field of industrial construction sites are used.
\end{abstract}

Keywords Extended Reality · Computer-Supported Cooperative Work (CSCW) • Augmented Reality · Virtual Reality · Collaboration

\section{Einleitung}

Ich denke, dass ein signifikanter Teil der Bevölkerung in den entwickelten Ländern und schließlich in allen Ländern jeden Tag AR-Erlebnisse haben wird, so selbstverständlich, wie drei Mahlzeiten am Tag zu essen. Es wird ein derart fester Bestandteil des Lebens werden. - Tim Cook, Vorstandsvorsitzender von Apple, 2016

Die Digitalisierung bietet eine große Vielfalt an verfügbaren Technologien, die Effizienzgewinne durch neue Formen der Zusammenarbeit und einen intuitiven Zu- 
gang zu Wissen versprechen. Bekannte Beispiele sind Kollaborationssysteme wie Microsoft Teams, Zoom oder Slack, die Videokonferenzen, Textnachrichten sowie Dateiaustausch ermöglichen. Andere Technologien werden zur Orchestrierung von Kundeninteraktionen oder Arbeitsabläufen eingesetzt. Organisationen, die mit dem digitalen Wandel Schritt halten wollen, stehen vor der Herausforderung, die für ihre Zwecke am besten geeigneten Kollaborationssysteme auszuwählen. Die Frage, die sich dabei stellt ist, wie diese Auswahl und das Verständnis über die Möglichkeiten vereinfacht werden kann.

Um die Auswahl zu erleichtern, befasst sich der Bereich ,Computer-Supported Cooperative Work' (CSCW) mit digitalen Informationssystemen zur Unterstützung von Organisationen in der Kollaboration. Diese Informationssysteme sind formale soziotechnische Systeme, die Informationstechnologien nutzen, um Informationen zu speichern, zu verarbeiten und zu verbreiten (Piccoli 2012, S. 21). Konventionelle Informationssysteme wurden zuletzt um Extended-Reality-Systeme (XRS) erweitert. Die Verwendung von XRS als kollaborative Systeme wird allgemein als eine der ,Killeranwendungen“ (Ens et al. 2019, S. 2) von XR angesehen.

Extended Reality (XR) ist ein Begriff, der sich auf alle realen und virtuellen kombinierten Umgebungen sowie Mensch-Maschine-Interaktionen bezieht, die durch Computertechnologie und tragbare Geräte erzeugt werden (Fast-Berglund et al. 2018, S. 32).

Die Technologien AR und VR, die bekanntesten XR-Technologien, stehen im Mittelpunkt dieser Arbeit. Dabei wird AR als System definiert, das 1) reale und virtuelle Inhalte kombiniert, 2) in Echtzeit interaktiv funktioniert und 3) in drei Dimensionen erfasst wird (Azuma 1997). VR beschreibt die Verwendung einer computergenerierten 3D-Umgebung - genannt Virtual Environment (VE) -, in der die Nutzer navigieren und möglicherweise interagieren können, was zu einer Echtzeitsimulation eines oder mehrerer der fünf Sinne des Nutzers führt (Guttentag 2010).

Die Forschung benennt die Dimensionen von XRS mit diversen Taxonomien, die versuchen, die Komplexität zu adressieren. Zum Beispiel kategorisieren Brockmann et al. (2013) relevante Taxonomien für kollaborative AR-Anwendungen in a) technisch orientierte Taxonomien, b) benutzerorientierte Taxonomien, c) informationsorientierte Taxonomien, d) interaktionsorientierte Taxonomien und e) ,Andere', jeweils mit eigenen Dimensionen. Brockmann et al. (2013) definieren daraus sechs Dimensionen, um XR zu beschreiben: 1. Raum, 2. Zeit, 3. Mobilität, 4. virtuelle Inhalte, 5. Rollenkonzept und 6. Visualisierungshardware. Die Verwendung vieler Dimensionen ist zwar umfassend, führt aber zu einer hohen Anzahl an möglichen Varianten. Wird diese Taxonomie als Beispiel genommen und auf Basis der zwei bis fünf Ausprägungen pro Dimension gerechnet, so führt dies zu 810 theoretisch möglichen Ergebnissen. Ziel dieser Arbeit ist, die Klassifikationsdimensionen in einer eigenen Taxonomie zu reduzieren, sowie eine nachvollziehbare Terminologie zu finden, um ein breiteres Verständnis und eine Operationalisierung von XRS zu ermöglichen.

Mit einer Kategorisierung wird eine Anleitung gegeben, wie in einem bestimmten Kollaborationsszenario XRS und entsprechende Alternativen berücksichtigt sowie implementiert werden sollen. Der Beitrag dieser Arbeit ist (1) die Kategorisierung, 
(2) die Terminologie und (3) die Illustrierung praktischer Implementierungen für XRS hinsichtlich des Aspekts der Kollaboration.

Dafür wird zuerst die Literatur zu XRS und Kollaboration betrachtet und basierend auf Literatur und Interviews, dann eine eigene CSCW-Matrix für XRS in Kollaborationsszenarien vorgeschlagen. Die Szenarien und jeweiligen XRS werden anhand von Illustrationen beschrieben und mit einer Diskussion zu Limitierungen der Taxonomie, sowie weiteren Forschungsthemen abgeschlossen.

\section{XRS und Kollaboration in der Literatur}

Kollaboration, Kommunikation sowie Kollaborationsmethoden sind eng miteinander verknüpft und werden als zusammenhängend betrachtet (Andriessen 2012). Eine Fülle von Forschungsarbeiten untersucht, wie Kollaborationssysteme die Zusammenarbeit (Lukosch et al. 2015) und die bestehende Kommunikation am Arbeitsplatz beeinflussen (Hofma et al. 2017).

Kollaboration kann als Interaktion von Teilnehmern beschrieben werden, die einer gemeinsamen Mission verpflichtet sind und bereit sind, das Wissen zu teilen, das zur Erfüllung dieser Mission notwendig ist (van Leeuwen und Fridqvist 2006). Kollaborationstechnologien sind der Hauptbestandteil der Informationsinfrastruktur, die die soziotechnische Technologie darstellt, in der Menschen, Systeme und Prozesse in einer kontinuierlichen Interaktion stehen (Nikas et al. 2007). Ein Ort, an dem die Kollaboration zwischen vielen Akteuren für eine gemeinsame Mission nötig ist, sind Baustellen. Aktivitäten auf Baustellen sind aufgrund der breiten Vielfalt an Akteuren und der klaren Mission, das entsprechende Konstrukt zu bauen, ein geeignetes Fallbeispiel, um solche Kollaborationsaktivitäten mithilfe von Kollaborationstechnologien $\mathrm{zu}$ veranschaulichen.

\subsection{Kategorisierung konventioneller Kollaborationsmethoden}

Einen Überblick über konventionelle Kollaborationsmethoden geben Bafoutsou und Mentzas (2002). Sie bezeichnen Kollaborationsmethoden als ,kollaborative Dienste', die von den folgenden, beispielhaften Tools bereitgestellt werden: E-Mail, Online Paging/Messaging, Chat, Whiteboard, Audio-/Videokonferenzen, Aufgabenliste, Kontaktmanagement, Bildschirmfreigabe, Umfragen/Abstimmungen, Besprechungsprotokolle/Aufzeichnungen, Tools zur Planung von Besprechungen, Projektmanagement und Dokumentenmanagement. Um dieses breite Spektrum an kollaborativen Systemen in kollaborative Szenarien einzuordnen, verwenden Bafoutsou und Mentzas das von Johansen (1988) skizzierte Vier-Quadrate-Zeit/Ort-Modell, das auch in dieser Arbeit zur Anwendung kommt.

Nach Penichet et al. (2007) basieren die meisten CSCW-Klassifikationen auf diesem ursprünglichen Modell von Johansen. Dies verdeutlicht, wie umfassend diese Klassifizierung ist. Andere Klassifikationen beziehen zusätzliche Dimensionen wie den Aufgabentyp und die Gruppengröße (Desanctis und Gallupe 1987) oder die Vorhersagbarkeit von Ort und Zeit mit ein (Grudin und Poltrock 2012). Dies führt zur bereits erwähnten Problematik der vielen theoretisch möglichen Ergebnisse. Das 
ursprüngliche Modell ermöglicht und erleichtert daher den Vergleich zwischen konventionellen und XR-basierten Kollaborationsmethoden in den jeweiligen Szenarien.

\subsection{Kategorisierung von XRS}

Für die Beschreibung von XR ist eines der am häufigsten verwendeten und zitierten Konzepte das Reality-Virtuality Continuum von Milgram et al. (1994). Dieses stellt einen Gestaltungsraum mit zwei Extremen dar: Das eine Extrem ist die physische Realität und das andere Extrem die Virtualität, eine vollständig computergenerierte virtuelle Umgebung. Dazwischen liegt das Mixed-Reality-Kontinuum. Innerhalb dieses Kontinuums sind AR und Augmented Virtuality (AV) zu finden. Das weniger bekannte AV beschreibt die Einbettung von realen Objekten in eine virtuelle Umgebung.

VR kann mit individuellen Headsets oder raumähnlichen immersiven VR-Umgebungen erzeugt werden und ist nach Milgram et al. (1994) nicht Teil des MixedReality-Kontinuums. Die genannte Technologie vermischt sich nicht mit der Realität, sondern erzeugt eine völlig eigenständige und fiktive Realität.

Es gibt verschiedene Ausprägungen der Technologien, die neben dem definierten Kontinuum platziert werden können. In der Literatur zu XR werden zusätzliche Dimensionen definiert, um zu differenzieren, z. B. technische Unterschiede und $\mathrm{Nu}$ ancierungen, die auf den Verwendungszweck, das Referenzobjekt oder die Menge der Nutzer zurückzuführen sind. Einige Taxonomien konzentrieren sich auf eine bestimmte Technologie wie AR (Azuma 1997) oder VR (Steuer 1992), während andere einen breiteren Ansatz verfolgen und AR mit VR kombinieren (Robinett 1994; Mann 2002).

Ens et al. (2019) definieren z.B. Zeit und Ort als relevante Dimensionen zur Klassifizierung von AR- und VR-Systemen inklusive eines direkten Verweises auf die CSCW-Time-Space-Matrix. Ihr Ziel ist es, alle erforderlichen Dimensionen zu erfassen, die in der Literatur diskutiert werden. Daher identifizierten sie neben Zeit und Ort die Dimensionen Symmetrie (Verhältnis von Rollen und Fähigkeiten der Kollaborateure), Künstlichkeit (von vollständig physischer bis zu vollständig digitaler Technologie), Fokus (das primäre Ziel der kollaborativen Aktivität) und Szenario (das Gesamtkonzept eines Systems gemäß Benutzer und Anwendungsfall).

Diese Dimensionen führen wiederum zu einer hohen Anzahl möglicher Ergebnisse von Kategorien und einer entsprechenden Komplexität. Daraus ergibt sich der Bedarf nach einer handhabbaren Kategorisierung, die umfassend ist, jedoch die Menge der Kollaborationsszenarien sowie die XRS-Varianten begrenzt.

\section{Einführung einer CSCW-Matrix für XRS}

Die Analyse bestehender Taxonomien zeigt zwei Ausprägungen auf, die konsolidiert werden. Entweder werden a) die Unterschiede in der Technologie beschrieben, ohne eine Anleitung für mögliche Anwendungsszenarien zu geben (wie bei Milgram et al. 1994), oder es wird b) eine umfassende Anzahl an Kategorisierungsdimensionen (wie bei Brockmann et al. 2013) gegeben, was die Komplexität erhöht und 


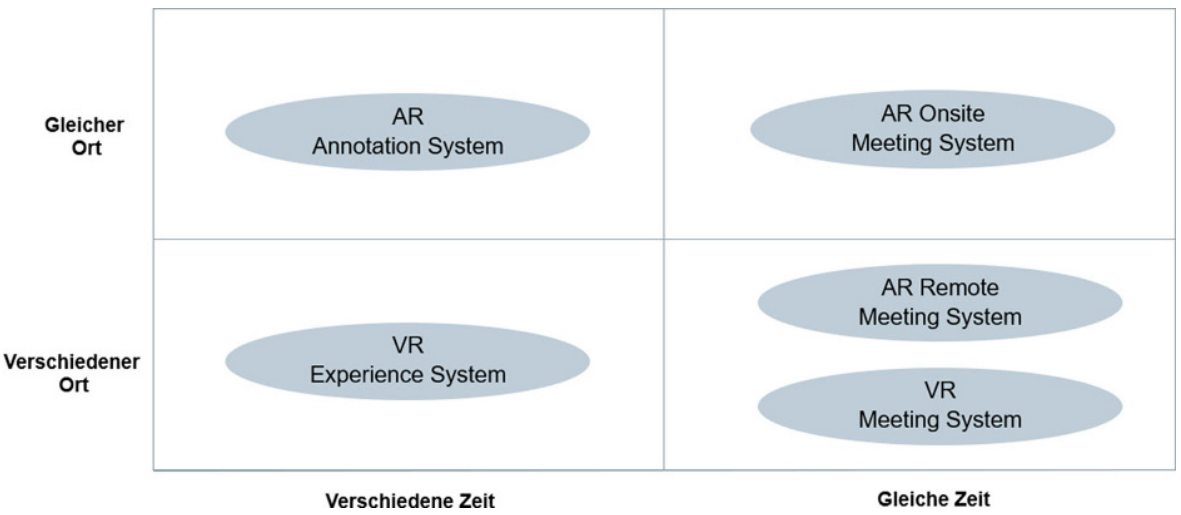

Abb. 1 CSCW-Matrix für XRS. (Eigene Darstellung)

einer intuitiven Anwendung in der Praxis entgegensteht. Durch die Fokussierung auf Zeit und Ort als relevante Dimensionen sowie durch die Einführung passender Terminologie soll eine verständliche Kategorisierung entstehen.

Basierend auf einer Literaturrecherche zu XR werden verschiedene Typen von XRS gesammelt und zu entsprechenden Begriffen zusammengefasst. Die Zuordnung zu den Szenarien wird dann in einem zweiten Schritt anhand der Illustrationen und verwandter Literatur verifiziert. Rajeb und Leclercq (2013) zum Beispiel nutzen ebenfalls die CSCW-Matrix, um eine Kategorisierung von Spatial Augmented Reality für Trainings vorzuschlagen.

Die Erläuterung der XRS-Typen basiert auf der hergeleiteten Terminologie und erfolgt in den jeweiligen Szenarien. Das Zuordnen von Kollaborationsmethoden, die auf XR-Technologien basieren, in die Vier-Quadrate-Karte ergibt die in Abb. 1 dargestellte Matrix.

Im Folgenden werden durch Illustrationen die jeweiligen Quadranten erläutert und aktuelle Methoden der Zusammenarbeit mit dem Potenzial von XRS exemplarisch auf Baustellen verglichen.

\subsection{XRS in komplexen Kollaborationsumgebungen - Anwendungen auf Baustellen}

Baustellen sind als Illustrationsszenarien besonders geeignet, da sie gekennzeichnet sind durch a) viele beteiligte Akteure, b) die ständige Veränderung der Umgebung, c) die Komplexität der Umgebung und d) die Notwendigkeit, über einen längeren Zeitraum zusammenzuarbeiten. Die Illustrationen sind aus Interviews mit sieben Bauexperten sowie mehreren Workshops abgeleitet. Diese Experten wurden im Rahmen eines Innovationsprojekts in einem DAX30-Konzern ausgewählt und haben sich bereiterklärt, freiwillig teilzunehmen. Alle Interviews, die in deutscher oder englischer Sprache geführt wurden, wurden aufgezeichnet und für die Analyse transkribiert. Die Experten sind z. B. Bauleiter, Inbetriebnahme-Ingenieure und technische Außendienstmitarbeiter, die auf Baustellen arbeiten oder bis vor kurzem gearbeitet haben. Des Weiteren wurden Anregungen aus der Literatur verwendet und 
dabei auch mögliche XR-Aktivitäten im Umfeld oder im Vorfeld einer Baustelleneinrichtung berücksichtigt, wie beispielsweise Planungsaktivitäten.

Ähnliche Bedingungen wie auf einer Baustelle können auch in anderen großen und komplexen Kollaborationsszenarien angetroffen werden, die viele Akteure sowie wechselnde Umweltbedingungen beinhalten, wie z. B. die Vorbereitung und Durchführung von Großveranstaltungen, das Management von Städten oder militärische, verteidigungspolitische und polizeiliche Aktivitäten.

\subsection{Kollaboration in Szenarien zur gleichen Zeit am gleichen Ort}

Der Großteil der Kollaborationen auf einer Baustelle findet in Echtzeit vor Ort statt. Diese persönlichen Interaktionen sind in der Regel nicht auf unterstützende Technik angewiesen, weswegen kollaborative Informationssysteme wie Whiteboards bisher nur eine untergeordnete Rolle spielen. Die Kommunikation von Angesicht zu Angesicht erfüllt bereits viele Anforderungen wie die Bereitstellung von Kontextinformationen und die Möglichkeit, vertrauliche Informationen auszutauschen.

XRS für solche Szenarien sind Augmented Reality Onsite Meeting Systems wie AR-Tischplatten, AR-Besprechungsräume oder AR-Applikationen für Wearables. Diese umfassen tragbare Computer wie Smartphones, Headsets oder Datenbrillen (Schattel et al. 2014) und lassen sich für kollaboratives Design, die Projektüberprüfung und Gruppenevaluationen nutzen (Rajeb und Leclercq 2013).

Beispielhaftes Szenario: Zwei Experten vergleichen die aktuelle Situation der Baustelle mit dem digitalen Modell der Planung, das der Umgebung digital überlagert ist. Ein AR-System kann dabei z.B. eine rechtzeitige Kollisionserkennung verschiedener Bauelemente bei Planungsfehlern ermöglichen. So wurde auch in den Interviews die Bedeutung von visuellen Informationen betont: „Bilder sind sehr wichtig, um Änderungen in Kundengesprächen besser erklären zu können. Wir verwenden Zeichnungen, Diagramme und alles andere, was es gibt“" (Inbetriebsetzer WB).

\subsection{Kollaboration in Szenarien zur gleichen Zeit an unterschiedlichem Ort}

Bei komplexen Bauvorhaben ist die Abstimmung mit Akteuren relevant, die nicht auf der Baustelle anwesend sind. Dies sind z. B. der Bauleiter oder die technische Abteilung. Eine dominierende Kollaborationsmethode für dieses Szenario sind Telefonate, die jedoch ihre Grenzen in Bezug auf die Erreichbarkeit des Gegenübers und die Kontextinformationen von der Baustelle haben.

XRS für solche Szenarien sind Augmented Reality Remote Meeting Systems. Dabei kann es sich um AR-Tischplatten mit Fernzugriff für die kollaborative Planung handeln (Shekhar Nittala et al. 2015) oder um die gemeinsame Nutzung von Grafikdokumenten und Anmerkungen in Echtzeit über Video-Streaming für sogenannten ,Remote Support‘, d.h. Fernunterstützung (Rajeb und Leclercq 2013).

Beispielhaftes Szenario: Ein Experte in der Zentrale oder auf einer anderen Baustelle unterstützt seinen Kollegen per Video und es wird ihm ermöglicht, virtuell in die Sichtlinie des lokalen Kollegen zu zeichnen, um ihn auf den nächsten Schritt der Aufgabe hinzuweisen. Der Experte kann außerdem virtuelle Bilder und Dokumente 
in das Sichtfeld einblenden, die den lokalen Kollegen bei der Problemlösung unterstützen. Wie im vorherigen Beispiel ist der große Vorteil die Visualisierung von Kontext- und erklärenden Informationen.

Auch Virtual Reality Meeting Systems können in diesen Szenarien verwendet werden. Ein Beispiel ist die Bauplanung direkt in einer immersiven Umgebung, die die Live-Zusammenarbeit unterstützt (Coppens und Mens 2018).

Beispielhaftes Szenario: Eine Gruppe von Experten trifft sich unabhängig von der lokalen Realität in einem virtuellen Raum, um Probleme zu diskutieren. Dieser virtuelle Raum kann durch einen lokalen Kontext angereichert werden, z. B. durch eine virtuelle Darstellung der Baustelle oder eine Planungsphase.

\subsection{Kollaboration in Szenarien zu verschiedenen Zeiten an verschiedenen Orten}

Aufgrund der vielen Akteure und des langen Zeitraums bei komplexen Baustellen muss ein erheblicher Teil der Kommunikation persistent dokumentiert werden. Dies geschieht z. B. über verschiedene Protokolle, Workflows und E-Mails. Diese Methoden bergen das Risiko einer unterschiedlichen Auslegung und Fehlinterpretation, was insbesondere dem Fehlen von Kontextinformationen geschuldet ist. XRS für solche Szenarien sind Virtual Reality Experience Systems.

Ein Beispielhaftes Szenario: Eine virtuelle Umgebung basiert auf einer digitalen Darstellung der Baustelle oder einer $360^{\circ}$-Aufnahme. Diese Umgebung kann Details über die geplante endgültige Konstruktion liefern oder die aktuelle Situation der Baustelle festhalten und damit den Status quo zu einem bestimmten Zeitpunkt aufzeigen. Den Experten wird ermöglicht, diese Umgebung orts- und zeitunabhängig zu kommentieren sowie zu aktualisieren und so eine Fülle von Informationen, Zukunftsszenarien und Kontextdetails bereitzustellen, um Fehlinterpretationen zu vermeiden (Casarin et al. 2018). Dies eignet sich auch zur Vorbereitung auf die Baustelle, wie von einem Gruppenleiter von Bauleitern erläutert: „Ein 3D Modell der Anlage in Virtual Reality ermöglichte dem Team, was auf die Anlage fährt, schon vorher quasi wie in einem Computerspiel durch die Anlage zu laufen. Effekt war ziemlich beeindruckend; wenn die Leute auf die Anlage gekommen sind haben sie keine Einführung mehr gebraucht, weil sie wussten sofort wo das Maschinenhaus ist, wo die Maschine steht, die Container und so weiter".

\subsection{Kollaboration in Szenarien zu verschiedenen Zeiten am gleichen Ort}

In dieser Kategorie spielt der Ort eine wesentliche Rolle bei der Zusammenarbeit. Es ist aber nicht notwendig oder möglich, dass alle beteiligten Akteure zum gleichen Zeitpunkt vor Ort sind. Die bereits erwähnten ortsgebundenen Kollaborationsmethoden, wie z. B. ein digitales schwarzes Brett, können auf Baustellen nicht angewendet werden, da verschiedene und wechselnde Orte von Bedeutung sind. In diesem Zusammenhang kann angenommen werden, dass die intuitivste Methode der Zusammenarbeit das Hinterlassen von Notizen oder Schildern am jeweiligen Ort ist. Dies ist wegen der rauen und unwirtlichen Umgebung auf Baustellen sowie der teils notwendigen Vertraulichkeit und Personalisierung nicht möglich. Die allgemeine 
Praxis ist daher, die Situation vor Ort z.B. in Mailberichten, mit Bildern oder per Telefonanruf zu beschreiben, was Potenzial für Fehlinterpretationen birgt.

XRS für solche Szenarien sind Augmented Reality Annotation Systems. Diese ermöglichen es, digitale Inhalte (z.B. 3D-Hologramme, Videos etc.) personalisiert, vertraulich, beständig und inhaltsreich an einem beliebigen Ort auf der Baustelle zu platzieren (Irlitti et al. 2013).

Beispielhaftes Szenario: Ein Bauleiter macht einen Überwachungsrundgang durch die Baustelle und überall dort, wo es ein zu behebendes Problem gibt, hinterlässt er eine digitale Annotation. Mit einem Knopfdruck hat er anschließend ein fertiges Protokoll und der markierte Bauarbeiter, der das Problem beheben muss, erhält einen Arbeitsauftrag. Der Bauarbeiter wird dann zu der Stelle navigiert und trifft auf die digitalen Annotationen des Bauleiters, die die zu erledigende Aufgabe eindeutig beschreiben und an der entsprechenden Stelle als AR-Hologramm, in der Luft schweben'. Dabei ist auch die einhergehende Dokumentierung der Aktivitäten von Relevanz. So wurde das Problem folgendermaßen beschrieben: „Und oft sagt der Kunde: ,Sie haben mir das letzte Mal dies und das versprochen“, und es ist nicht mehr dokumentiert" (Inbetriebsetzer WB).

\section{Diskussion und Ausblick}

Die Ausarbeitung zeigt eine praxisnahe und handhabbare Kategorisierung, die mit einer strukturierten Terminologie XRS beschreibt. Dies soll der Fragestellung gerecht werden, die Auswahl und das Verständnis von XRS-Kollaborationssystemen zu erleichtern. Weitere Dimensionen, die in der Literatur identifiziert wurden (z. B. Ens et al. 2019), blieben bewusst unberücksichtigt. Die Terminologie für die jeweiligen XRS wurde im Rahmen der Forschung zu diesem Konzeptpapier festgelegt. Dabei hätte es andere Möglichkeiten der Formulierung gegeben. Es kann nun analysiert werden, ob die Zielstellung erreicht wird, dass ein solcher Ansatz das Verständnis und die Auswahl von XRS in der Managementpraxis unterstützt. Dies kann mit Hilfe der Matrix nun in einer systematischen Weise erfolgen, zum Beispiel indem die Organisation 1) ihre aktuellen Methoden und Werkzeuge je Szenario beschreibt, 2) die aktuellen Schwierigkeiten und Anforderungen in den Szenarien auflistet und 3) diese den potenziellen XRS und deren Möglichkeiten gegenüberstellt.

Limitationen des Modells sind die Heterogenität der XRS und die Einsatzmöglichkeiten. Fast alle XRS-Typen könnten in jedes der Szenarien eingebaut werden. Ein Beispiel ist die Dollhouse-VR/AR-Lösung von Ibayashi et al. (2015), die eine AR-Ansicht auf eine Tischplatte bietet. Sie ermöglicht sowohl den Blick in einen künstlichen Raum als auch eine VR-Ansicht von der Position innerhalb des künstlichen Raums und kombiniert so AR und VR sowie die Zusammenarbeit vor Ort und aus der Ferne (Ibayashi et al. 2015). Daher sind einige XRS für mehr als eines der Kollaborationsszenarien geeignet und die vorgestellte CSCW-Matrix für XRS kann zur Klassifizierung verschiedener Funktionalitäten verwendet werden (Penichet et al. 2007).

Ein weiteres Beispiel sind georeferenzierte AR-Annotationen, die in einer synchronen Vor-Ort-Kollaboration erstellt und aufgrund ihrer Persistenz auch zu einem 
späteren Zeitpunkt verwendet werden können. Insbesondere im Szenario ,Gleicher Ort zu verschiedenen Zeiten' sind die aktuellen Kollaborationsmethoden unzureichend. Um Informationen über einen bestimmten Zeitraum hinweg an einem bestimmten Ort zu vermitteln, sind diese Möglichkeiten entweder abhängig von teurer Hardware oder anfällig für Umwelteinflüsse sowie öffentlich sichtbar. Hardware wie Bildschirme können nicht an beliebigen Orten installiert werden und nichttechnische Lösungen wie Schilder und Post-its sind in der Darstellung auf 2D limitiert und nicht personalisiert. Aufgrund der fehlenden konventionellen Kollaborationsmethoden für georeferenzierte, asynchrone Kollaborationsszenarien ist es beachtlich, dass diese Anwendung für XRS in der Forschung bisher wenig thematisiert wurde (de Belen et al. 2019).

Die aktuelle Geschwindigkeit der technologischen Entwicklung für XRS sorgt für eine dynamische Entwicklung von Attributen, Merkmalen und Funktionen sowie für die zunehmende Verschmelzung von AR und VR. Das bedeutet, dass in zukünftigen XRS ein nahtloses Hin und Her auf dem Kontinuum zwischen Realität und Virtualität ermöglicht werden könnte (Benford et al. 2001). Es bleibt abzuwarten, ob eine Taxonomie allgemein genug sein kann, um ihre Gültigkeit langfristig zu behalten.

Eine weitere Vereinfachung ist die Vier-Felder-Matrix selbst, da sie Zeit und Ort nur nach den Instanzen von gleich und verschieden kategorisiert. Ein Kontinuum von Technologien zu haben, rechtfertigt es, ein Kontinuum der Dimensionen einzuführen, um die Zusammenarbeit zu optimieren, z. B. anhand der Wichtigkeit von Ort (im Sinne der Kontextinformationen) und Zeit (im Sinne von gleichzeitigem Arbeiten). Um dabei das geeignetste XRS zu wählen, ist ein bedeutsamer Faktor die Transaktionskosten für die Realisierung einer bestimmten Kollaborationsmethode (Inkpen 1996). Insbesondere Reisekosten und -zeit können die Möglichkeit der Kollaboration am gleichen Ort unterbinden.

Um das am besten geeignete XRS auszuwählen, sind weitere Schritte erforderlich wie das Verstehen der Motive zur Nutzung von XRS im jeweiligen Anwendungsfall. Die Motive, die VR und AR antreiben, werden in der Forschung gemäß Steffen et al. jedoch nicht ausreichend adressiert (Steffen et al. 2019) und werden auch in der vorgeschlagenen Matrix nicht berücksichtigt. Die vermeintliche Überlegenheit von Extended-Reality-Technologien basiert zu einem bestimmten Grad auf den umfangreichen Möglichkeiten der Darstellung. Komplexe Zusammenhänge, die nicht bereits in den zugrundeliegenden Daten berücksichtigt werden, können auch nicht visualisiert werden und damit wird dieser vermeintliche Vorteil unbrauchbar. Allerdings können XRS von der Nützlichkeit anderer Technologien wie dem Internet der Dinge profitieren, da sie als intuitive Schnittstelle zur Fülle an verfügbaren Daten dienen können (White et al. 2019). Zusammenfassend soll mit der CSCW-Matrix für XRS und der enthaltenen Terminologie die Wahl von geeigneten XRS für die Kollaboration in einem Ort-Zeit-Kontext vereinfacht werden.

Funding Open Access funding enabled and organized by Projekt DEAL.

Open Access Dieser Artikel wird unter der Creative Commons Namensnennung 4.0 International Lizenz veröffentlicht, welche die Nutzung, Vervielfältigung, Bearbeitung, Verbreitung und Wiedergabe in jeglichem Medium und Format erlaubt, sofern Sie den/die ursprünglichen Autor(en) und die Quelle ordnungsgemäß nennen, einen Link zur Creative Commons Lizenz beifügen und angeben, ob Änderungen vorgenommen wurden. 
Die in diesem Artikel enthaltenen Bilder und sonstiges Drittmaterial unterliegen ebenfalls der genannten Creative Commons Lizenz, sofern sich aus der Abbildungslegende nichts anderes ergibt. Sofern das betreffende Material nicht unter der genannten Creative Commons Lizenz steht und die betreffende Handlung nicht nach gesetzlichen Vorschriften erlaubt ist, ist für die oben aufgeführten Weiterverwendungen des Materials die Einwilligung des jeweiligen Rechteinhabers einzuholen.

Weitere Details zur Lizenz entnehmen Sie bitte der Lizenzinformation auf http://creativecommons.org/ licenses/by/4.0/deed.de.

\section{Literatur}

Andriessen JHE (2012) Working with groupware: understanding and evaluating collaboration technology. Springer,

Azuma RT (1997) A survey of augmented reality. Found Trends Human Comput Interact 8(2-3):73-272. https://doi.org/10.1561/1100000049

Bafoutsou G, Mentzas G (2002) Review and functional classification of collaborative systems. Int J Inf Manage 22(4):281-305. https://doi.org/10.1016/S0268-4012(02)00013-0

de Belen RAJ, Nguyen H, Filonik D, Del Favero D, Bednarz T (2019) A systematic review of the current state of collaborative mixed reality technologies: 2013-2018. AIMS Electron Electr Eng 3(2):181-223. https://doi.org/10.3934/ElectrEng.2019.2.181

Benford S, Greenhalgh C, Rodden T, Pycock J (2001) Collaborative virtual environments. Commun ACM 44(7):79-85. https://doi.org/10.1145/379300.379322

Brockmann T, Krueger N, Stieglitz S, Bohlsen I (2013) A framework for collaborative augmented reality applications. 19th Americas Conference on Information Systems, AMCIS 2013-Hyperconnected World: Anything, Anywhere, Anytime (1:1986), S 83-92

Casarin J, Pacqueriaud N, Bechmann D (2018) UMI3D: a unity3D toolbox to support CSCW systems properties in generic 3D user interfaces. Proceedings of the ACM on Human-Computer Interaction (2:CSCW).. https://doi.org/10.1145/3274298

Coppens A, Mens T (2018) Towards collaborative Immersive environments for parametric modelling. Lecture notes in computer science (including subseries lecture notes in artificial intelligence and lecture notes in bioinformatics), Bd. 11151 LNCS. Springer, Berlin Heidelberg https://doi.org/10. 1007/978-3-030-00560-3_44

DeSanctis G, Gallupe RB (1987) A foundation for the study of group decision support systems. Management science 33(5):589-609

Ens B, Lanir J, Tang A, Bateman S, Lee G, Piumsomboon T, Billinghurst M (2019) Revisiting collaboration through mixed reality: the evolution of groupware. Int J Hum Comput Stud. https://doi.org/10.1016/ j.ijhes.2019.05.011

Fast-Berglund Å, Gong L, Li D (2018) Testing and validating extended reality (XR) technologies in manufacturing. Procedia Manuf 2018(25):31-38. (https://www.sciencedirect.com/science/article/pii/ S2351978918305730/pdf?md5=0e2edecf9920c077e3dd6be3d0e262b6\&pid=1-s2.0-S23519789183 05730-main.pdf)

Grudin J, Poltrock SE (2012) Taxonomy and theory in computer supported cooperative work. In: The oxford handbook of organizational psychology, Bd. 2, S 1323-1348 https://doi.org/10.1093/oxfordhb/ 9780199928286.013.0040

Guttentag DA (2010) Virtual reality: applications and implications for tourism. Tour Manag 31(5):637-651. https://doi.org/10.1016/j.tourman.2009.07.003

Hofma CC, Avital M, Jensen TB (2017, December) Liquid workplaces: The potential implications of virtual reality on the workplace. In: The 40th Information Systems Research Conference in Scandinavia, vol 8. IRIS, pp 31-43

Ibayashi H, Sugiura Y, Sakamoto D, Miyata N, Tada M, Okuma T, Kurata T, Mochimaru M, Igarashi T (2015) Dollhouse VR: a multi-view, multi-user collaborative design workspace with VR technology. SIGGRAPH Asia 2015 Posters, SA 2015, S 2-3 https://doi.org/10.1145/2820926.2820948

Inkpen AC (1996) Creating knowledge through collaboration. Calif Manage Rev. https://doi.org/10.2307/ 41165879

Irlitti A, Von Itzstein S, Alem L, Thomas B (2013) Tangible interaction techniques to support asynchronous collaboration. IEEE International Symposium on Mixed and Augmented Reality 2013 (October 2013). IEEE, 
Johansen R (1988) Groupware: computer support for business teams. The Free Press,

van Leeuwen JP, Fridqvist S (2006) An information model for collaboration in the construction industry. Comput Ind 57(8-9):809-816. https://doi.org/10.1016/j.compind.2006.04.011

Lukosch S, Billinghurst M, Alem L, Kiyokawa K (2015) Collaboration in augmented reality. Comput Support Coop Work CSW Int J 24(6):515-525. https://doi.org/10.1007/s10606-015-9239-0

Mann S (2002) Mediated reality with implementations for everyday life. In: Presence: teleoperators and virtual environments, S 1-13 (http://www.mitpressjournals.org/toc/pres/11/4)

Milgram P, Takemura H, Utsumi A, Kishino F (1994) Augmented reality: a class of displays on the realityvirtuality continuum. In: Systems research (2351:Telemanipulator and Telepresence technologies), S 282-292

Nikas A, Poulymenakou A, Kriaris P (2007) Investigating antecedents and drivers affecting the adoption of collaboration technologies in the construction industry. Autom Constr 16(5):632-641. https://doi. org/10.1016/j.autcon.2006.10.003

Nittala SA, Li N, Cartwright S, Takashima K, Sharlin E, Sousa MC (2015) PLANWELL: spatial user interface for collaborative petroleum well-planning. SIGGRAPH Asia 2015 Mobile Graphics and Interactive Applications, SA 2015 (November). https://doi.org/10.1145/2818427.2818443

Penichet VMR, Marin I, Gallud JA, Lozano MD, Tesoriero R (2007) A classification method for CSCW systems. In: Electronic notes in theoretical computer science (168:SPEC. ISS.)., Elsevier, S 237-247 https://doi.org/10.1016/j.entcs.2006.12.007

Piccoli G (2012) Information systems for managers: texts and cases. Wiley,

Rajeb SB, Leclercq P (2013) Using spatial augmented reality in synchronous collaborative design. Lecture notes in computer science (including subseries lecture notes in artificial intelligence and lecture notes in Bioinformatics, Bd. Vol. 8091 LNCS. https://doi.org/10.1007/978-3-642-40840-3_7

Robinett W (1994) Synthetic experience. In: State of the art in computer graphics, S 177-194 https://doi. org/10.1007/978-1-4612-4306-9_9

Schattel D, Tonnis M, Klinker G, Schubert G, Petzold F (2014) On-site augmented collaborative architecture visualization. ISMAR 2014-IEEE International Symposium on Mixed and Augmented Reality-Science and Technology 2014, Proceedings, S 369-370 https://doi.org/10.1109/ISMAR.2014. 6948493

Steffen JH, Gaskin JE, Meservy TO, Jenkins JL, Wolman I (2019) Framework of affordances for virtual reality and augmented reality. J Manag Inf Syst 36(3):683-729. https://doi.org/10.1080/07421222. 2019.1628877

Steuer J (1992) Defining virtual reality: dimensions determining telepresence. J Commun 42(4):73-93. https://doi.org/10.1111/j.1460-2466.1992.tb00812.x

White G, Cabrera C, Palade A, Clarke S (2019) Augmented reality in IoT. Lecture notes in computer science (including subseries lecture notes in artificial intelligence and lecture notes in bioinformatics, Bd. 11434 LNCS, S 149-160 https://doi.org/10.1007/978-3-030-17642-6_13 